\title{
Spectrum of Cholangiocarcinoma with Special Emphasis on Treatment Modalities and Outcome at a Rural Tertiary Care Centre
}

\author{
Dwaipayan Samaddar1, Duraisamy Ravichandran Rajan², Jaya Bagchi Samaddar³, Gautam Das ${ }^{4}$ \\ ${ }^{1}$ Department of General Surgery, North Bengal Medical College and Hospital, Kolkata, West Bengal, India. ${ }^{2}$ Department \\ of Surgery, North Bengal Medical College and Hospital, Kolkata, West Bengal, India. ${ }^{3}$ Department of Pathology, \\ Islampur Sub Divisional Hospital, West Bengal, India. ${ }^{4}$ Department of Surgery, Institute of Post Graduate Medical \\ Education and Research, Kolkata India.
}

\section{ABSTRACT}

\section{BACKGROUND}

Cholangiocarcinoma (CCA) is a heterogeneous group of aggressive malignancies emerging from the canals of Hering to the CBD. CCA are rare tumours accounting for $3 \%$ of gastrointestinal tumours with an incidence of $<2 / 100000$, with an increased incidence in Asia. They present late and have a grave prognosis. The only curative option is surgical resection. The aim was to study the demographic and clinical spectrum of CCA with special emphasis on practicable diagnostic modalities, treatment strategies and their outcome at our rural tertiary setup.

\section{METHODS}

This was a single centre observational study with a longitudinal design conducted over a period of 2 years. 30 patients presenting with features or diagnosis of CCA to the Department of General Surgery and the Department of Radiotherapy of North Bengal Medical College and Hospital, were studied with special reference to treatment and outcome. Data was collected, compiled on Excel sheet and analysed using GraphPad QuickCalcs 2018 (San Diego, CA).

\section{RESULTS}

Mean age of the patients was 52.36 years with a female: male ratio $1.5: 1$. They presented mainly with jaundice (90\%), clay coloured stool (83.3\%), pruritus (66.6\%), anaemia (56.6\%), abdominal lump (43.3\%) and pain abdomen $(33.6 \%)$ reflecting a late presentation. Diagnosis and staging were done by blood parameters and available imaging studies in our setup (abdominal ultrasound, CECT and MRCP). The most common type was Distal CCA (60\%) followed by Perihilar CCA (33.3\%) and Intra-hepatic CCA (6.6\%). Pancreaticoduodenectomy was done in $26.6 \%$ of patients and resection with hepaticojejunostomy in $10 \%$ of patients; surgical biliary bypass in $20 \%$ of patients, chemotherapy in $6.6 \%$ and PTBD followed by chemotherapy in $36.6 \%$. After 1 year $72.7 \%$ of those who underwent curative surgery were having disease free life and $26.3 \%$ of those who underwent palliative treatment were having improved quality of life.

\section{CONCLUSIONS}

Early detection and surgery may offer an improved survival and disease free life in CCA even in a modest rural tertiary care setup.

\section{KEY WORDS}

Cholangiocarcinoma, Pancreatoduodenectomy, Hepaticojejunostomy
Corresponding Author: Dr. Jaya Bagchi Samaddar, DB-12/123, Sector 1 , Saltlake City, Kolkata-700064, West Bengal, India.

E-mail: jbsam_ae@yahoo.com

DOI: $10.14260 / j e m d s / 2020 / 296$

Financial or Other Competing Interests: None.

How to Cite This Article:

Samaddar D, Rajan DR, Samaddar JB, et al. Spectrum of cholangiocarcinoma with special emphasis on treatment modalities and outcome at a rural tertiary care centre. J. Evolution Med. Dent. Sci. 2020;9(16):1359-1364, 10.14260/jemds/2020/296

Submission 18-02-2020, Peer Review 04-04-2020, Acceptance 10-04-2020, Published 20-04-2020.

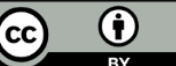




\section{BACKGROUND}

Cholangiocarcinoma (CCA) is a heterogeneous group of malignancies that can emerge from the canals of Hering to the main bile duct. Worldwide CCA accounts for approximately $3 \%$ of all gastrointestinal tumours and has an overall incidence $<1-2 / 100,000$.[1] Epidemiologic studies suggest that its incidence has been increasing in Western countries during the last decades. ${ }^{[1,2]}$ In the last three decades, a trend has been observed towards an older patient age at the time of diagnosis (median range of 65 to 70 years)and a relative increase in the ratio of intrahepatic $\mathrm{CCA}(\mathrm{IH}-\mathrm{CCA})$ to extrahepatic CCA(EH-CCA). IH-CCA are the tumours arising from biliary epithelium in intrahepatic bile ducts above the level of the left and right main ducts.[3] EH-CCA can further be subdivided into perihilar CCA ( $\mathrm{p}$-CCA,also called Klatskin tumours), and distal tumours (d-CCA).[4] CCA accounts for about $20 \%$ of the deaths from hepatobiliary cancers and $13 \%$ of the total cancer mortality worldwide. CCA is one of the most fatal cancers: although 1-year mortality has improved over time, the 5 -year survival is still as low as $10 \% .{ }^{[5]}$ SEER analysis of 2010-11 revealed that the highest incidence in Asians is most likely due to environmental and genetic differences.[6] The highest incidence rates of IH-CCA are reported in northern Thailand (up to 113 per 100,000 person-years in men and 50 per 100,000 person-years in women), where the liver fluke Opisthorchis viverrini is endemic. [7]

The vast majority of CCA $(70 \%)$ occur sporadically without any apparent cause. However there are certain recognised risk factors for this tumour, such as congenital biliary anomalies like choledochal cyst, primary sclerosing cholangitis (PSC), hepatolithiasis, parasitic infections, chronic typhoid carriage, bile duct adenoma, biliary papillomatosis, drug exposure and genetic risks.[8] Chronic biliary inflammation is the common denominator in these conditions. An inflammatory milieu is believed to dysregulate or change the expression patterns of growth factors, proinflammatory cytokines and their receptors. Cytokines produced by cholangiocytes and activated macrophages can modulate gene expression and lead to activation of carcinogen metabolism. KRAS modulation is seen more in pCCA whereas BAP1,IDH1/2 seen more in non-liver fluke CCA. TP53, SMAD4 are mainly associated with liver fluke induced CCA. Next Generation Sequencing suggested that dysregulation of GFR, AKT, VEGF, PDGF, FDGF, IL-6 is responsible for CCA thus reflecting the combination of inflammation, proto oncogene activation and tumour suppressor gene inactivation in its causation. [9]

Based upon their macroscopic growth pattern, CCA are classified as mass-forming, periductal-infiltrating or intraductal-papillary. IH-CCA are predominantly massforming, while EH-CCA are typically periductal-infiltrating. Histopathologically, $90 \%$ to $95 \%$ of CCA are adenocarcinomas of moderate to poor differentiation, with characteristic mucin expression and highly desmoplastic stroma.[10,11] CK7 and CK19 expression are characteristic of CCA, but both proteins can also be expressed in hepatocellular carcinoma (HCC) and metastatic adenocarcinomas.

The clinical presentation depends on the stage and location of the tumour. As there are no specific symptoms in the early stage many present in an advanced stage of the disease. Patients with EH-CCA usually presents with painless progressive obstructive jaundice, whereas those with IH-CCA usually presents with abdominal pain. Common complaints include dark urine, pruritus, clay coloured stools, weight loss and fever.

Diagnosis is usually made upon clinical presentation and investigations (blood, abdominal ultrasound, CECT, MRCP). Although the rapid development of imaging technology and instrumentation has enabled the accurate demonstration of lesions, these are of limited values in early CCA where there are small or no changes in morphology. These can be addressed to by the use of cytology or tissue biopsy via ERCP, PTC, cholangioscopy or EUS-FNA. In our setup diagnosis is based on clinical presentation and non-invasive imaging (USG abdomen, CECT and MRCP).CECT visualizes the local anatomical structures, measures the size of the tumour and the extent of the bile duct dilatation and detects regional lymphadenopathy, lobar atrophy and satellite nodules. It also does precise multidirectional assessment of biliary and vascular involvement, which helps in the accurate prediction of resectability. ${ }^{[12]}$ Similarly MRCP in combination with MRI is a reliable non-invasive diagnostic method for the pretherapeutic staging of CCA. Due to its intrinsic high tissue contrast and multiplanar ability, MRI with MRCP is capable of examining all the structures involved (bile ducts, vessels, hepatic parenchyma). A precise preoperative assessment of the tumour can therefore be achieved.[13] A dilated extrahepatic bile duct terminating abruptly at its distal aspect without a concomitantly dilated pancreatic duct suggests a distal CCA. In patients with a stricture of the distal bile duct and a clinical presentation consistent with CCA, histologic confirmation of malignancy is generally unnecessary, unless non-operative therapy is planned.[14]

The most commonly used tumour marker for CCA is CA 19-9. Its accuracy in distinguishing IH-CCA from HCC is $63 \%$ to $67 \% .{ }^{[15]}$ Currently, there are three major staging systems for IH-CCA: (1) the American Joint Committee on Cancer/Union for International Cancer Control (AJCC/UICC); (2) the Liver Cancer Study Group of Japan (LCSGJ); and (3) the National Cancer Center of Japan (NCCN) staging systems. The AJCC/UICC staging system has shown stage-survival correlation, but it is limited by its requirement for histology to determine Tis and T4.[16]

The Bismuth-Corlette classification was developed to guide surgical therapy for pCCA but it is not a staging system per se: Type I - Neoplasm arises near the biliary confluence without involvement of the left and right biliary ducts, Type II Neoplasm arises at the biliary confluence with extension to left and right ducts, Type IIIa Neoplasm arises at the biliary confluence with extension to the right hepatic duct up to the second order ducts, Type IIIb Neoplasm arises at the biliary confluence with extension to left biliary duct up to the second order ducts, Type IV Neoplasm arises at the biliary confluence with bilateral extension to the second order biliary ducts. The two most commonly used staging systems include the AJCC/UICC and the Memorial Sloan-Kettering Cancer Center (MSKCC) staging systems. In the most current seventh edition of the AJCC/UICC staging system, pCCA and dCCA are for the first time staged separately; thus, it requires further validation of its prognostic value. The MSKCC staging system failed to stratify resectable from unresectable patients 
with sufficient accuracy. New staging systems have been proposed awaiting further validation.[17]

The seventh edition of the AJCC/UICC is currently the only staging system for dCCA. Its use is limited by a lack of correlation of its T-stages to outcomes after resection, and the need for microscopic evaluation of tumour invasion depth.[18]

Surgical resection is the preferred treatment for CCA. Contraindications to surgical resection include bilateral, multifocal disease, distant metastases and comorbidities associated with operative risks outweighing the expected benefits of a surgery. PSC patients with pCCA should preferentially be treated with liver transplantation due to the field defect in PSC and frequent underlying advanced fibrosis. Dependent on the tumour extent, IH-CCA are resected by segmentectomy with $78 \%$ to $82 \%$ of patients requiring major segmentectomy ( $>3$ segments).[10,11] In cases in which low volume of the remaining hepatic lobe is prohibitive to resection, preoperative portal vein embolization of the ipsilateral hepatic lobe can result in compensatory hypertrophy of the contralateral lobe, thereby, permitting subsequent hemihepatectomy. While resection of BismuthCorlette type IV perihilar CCA is not considered an absolute contraindication to resection, neoadjuvant chemoradiation followed by orthotopic liver transplantation (OLT) is the preferable treatment based upon its excellent outcomes. Bismuth-Corlette type 1,2 are sometimes amenable to segmental resection and hepaticojejunostomy. Distal CCA are most commonly resected by conventional or pyloruspreserving pancreaticoduodenectomy with lymphadenectomy. pCCA tend to invade the right hepatic artery (RHA) due to its proximity. Recently, successful RHA resection and reconstruction in pCCA patients has been reported with perioperative mortality rates of less than $5 \%$ and 5-year survival rates of up 30\%.[19] In selected patients with portal vein or local invasion, pancreaticoduodenectomy with portal vein resection or hepatopancreaticoduodenectomy has been performed at specialized centers with R0 resection rates of $74 \%$ to $85 \%$ and 5 -year survival rates of $11 \%$ to $37 \%$; however, it is limited by high morbidity and mortality rates.[20]

Major postoperative complications include intraabdominal abscesses and bile duct leaks.[10,21,22,23] Preoperative biliary drainage in patients with malignant jaundice is an area of controversy. Uncorrected preoperative jaundice has been associated with higher rates of postsurgical abscesses, bile leaks, biliary fistulae and sepsis. However, several studies failed to show significant improvements with presurgical biliary drainage, and some reported increased overall and postsurgical complication rates. Pre surgical biliary drainage should be pursued in patients with a bilirubin of $>10 \mathrm{mg} / \mathrm{dL}$, cholangitis, neoadjuvant treatment and delayed surgery.[24,25]

Lymph node metastases and R0 status are the two major prognostic factors influencing outcomes after resection.[10,14,26] Approximately 45\% of patients undergoing resection are found to be $\mathrm{N}+.{ }^{[27]}$ Five-year survival of $\mathrm{N}+$ versus N0 disease is $0 \%$ to $9 \%$ versus $36 \%$ to $43 \%$ in iCCA, $0 \%$ to $29 \%$ versus $32 \%$ to $67 \%$ in pCCA, and $16 \%$ to $21 \%$ versus $42 \%$ to $61 \%$ in dCCA. ${ }^{[14,28,29]}$ Based on a recent metaanalysis, NCCN recommends adjuvant chemotherapy with fluoropyrimidine or gemcitabine based regimens for patients with R1-resection and/or N1 disease.[30] We studied the demographic and clinical spectrum of CCA with special emphasis on their diagnostic and treatment strategies and outcome at our rural tertiary setup.

\section{METHODS}

\section{Study Setting and Design}

This is an institution based observational study with longitudinal study design. 30 consecutive patients presenting with features or diagnosis of CCA to the Department of General Surgery and the Department of Radiotherapy in North Bengal Medical College and Hospital were studied for two years (May 2017- April 2019).

\section{Methodology}

This study was conducted after approval from institutional ethical committee. Written consent was taken, confidentiality ensured and the data was purely utilised for academic purpose. A detailed clinical history was collected from each patient using a preformed data collection sheet, then clinically examined and investigated thoroughly. Patients were provided with standard treatment modality available at our rural tertiary care hospital according to their stage of disease, which included surgical intervention (curative/palliative), chemotherapy and PTBD available at our rural tertiary care hospital. The patients were followed up for the period of hospital stay and also after 2 weeks postsurgical resection and then in an interval of 3 months for the study period.Clinical improvement was defined as alleviation of jaundice, pruritus and pain and weight gain.

\section{Statistical Analysis}

Data was collected and compiled in Excel sheet followed by analysis using Graph Pad Software and Graph Pad Quick Calcs 2018 (San Diego, CA). Descriptive statistical analysis included the calculation of means and standard deviation (SD) of the data obtained. Continuous variables were expressed as mean \pm SD and compared using a two tailed Student's paired $t$ test. Categorical data was analysed using chi square test. A p value $<0.05$ was considered statistically significant.

\section{RESULTS}

The incidence was more common in the age groups from 41 to 50 years (33.3\% of patients) and 51 to 60 years $(30 \%$ of patients). The minimum age of occurrence of CCA in this study was 28 years and maximum was 72 years. Mean age was 52.36 years (SD of 10.25). Though the age group of EHCCA was lesser than IH-CCA it was not significant $(\mathrm{p}=0.4485)$.

There were $12(40 \%)$ males and $18(60 \%)$ females (a female: male ratio of 1.5: 1). There was no significant gender distribution amongst IH-CCA and EH-CCA groups $(\mathrm{p}=0.654)$. The most common presenting feature was jaundice(90\%) followed by clay colored stool (83.3\%), pruritus (66.6\%), weight loss (46.6\%), abdominal lump (43.3\%), pain abdomen 
(33.3\%), fever with chills and rigor (23.3\%), tenderness $(23.3 \%)$ and ascites (20\%).

Among the study population of 30 patients, $2(6.6 \%)$ had IH-CCA, 10 (33.3\%) p-CCA and 18 (60\%) d-CCA. Histologically all were adenocarcinomas. $11(36.6 \%)$ patients were offered curative treatment and 19 (63.2\%) palliative treatment. In the curative treatment group, Pancreaticoduodenectomy was done in $8(26.6 \%)$ patients and Resection with Hepaticojejunostomy in $3(10 \%)$ patients. In the palliative treatment group, surgical internal bypass was done in $6(20 \%)$ patients, chemotherapy given to 2 $(6.6 \%)$ patients and $11(36.6 \%)$ were provided with PTBD and then chemotherapy. (table 1)

\begin{tabular}{|c|c|c|c|c|c|}
\hline Type & $\mathbf{n}$ & Mean \pm SD & t & & p \\
\hline IH-CCA & 2 & $56 \pm 12.73$ & \multirow{2}{*}{1.1765} & \multirow{2}{*}{\multicolumn{2}{|c|}{0.4485}} \\
\hline EH-CCA & 28 & $36 \pm 11.31$ & & & \\
\hline \multicolumn{6}{|c|}{ Table 1. Mean Age Distribution among IH-CCA \& EH-CCA } \\
\hline \multicolumn{3}{|c|}{ Treatment } & Frequency & \multicolumn{2}{|c|}{ Percentage (\%) } \\
\hline \multirow[b]{2}{*}{$\begin{array}{l}\text { Curative } \\
(\mathrm{n}=11)\end{array}$} & Pancreati & lenectomy & 8 & 26.6 & \multirow[b]{2}{*}{$\begin{array}{c}36.6 \\
(n=11)\end{array}$} \\
\hline & $\begin{array}{r}\text { Res } \\
\text { Hepati }\end{array}$ & $\begin{array}{l}\text { with } \\
\text { ostomy }\end{array}$ & 3 & 10 & \\
\hline \multirow{4}{*}{$\begin{array}{l}\text { Palliative } \\
(\mathrm{n}=19)\end{array}$} & Surgical & al bypass & 6 & 20 & \multirow{3}{*}{$\begin{array}{c}63.2 \\
(n=19)\end{array}$} \\
\hline & Che & & 2 & 6.6 & \\
\hline & PTBD follow & hemotherapy & 11 & 36.6 & \\
\hline & Total & & 30 & & 00 \\
\hline \multicolumn{6}{|c|}{ Table 2. Type of Treatment Offered to Patients $(n=30)$} \\
\hline
\end{tabular}

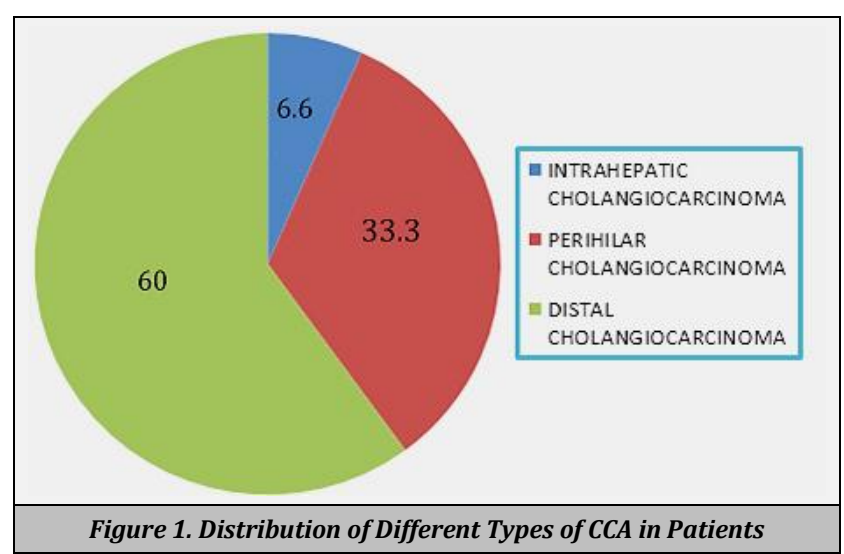

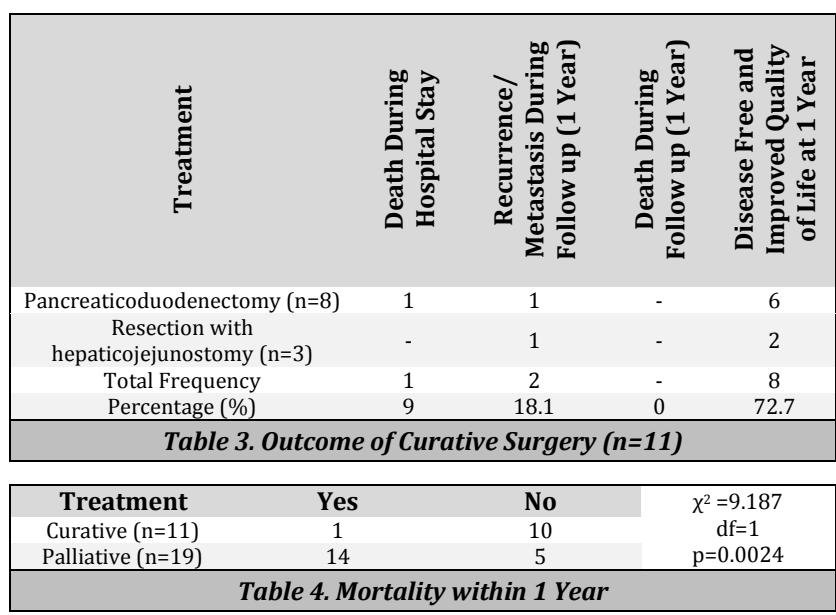

25(83.3\%) patients clinically improved after treatment. Among the patients to whom curative treatment $(\mathrm{n}=11)$ was given, 10 (90.9\%); and to whom palliative treatment $(n=19)$ was given, $15(78.9 \%)$ were clinically improved $(\mathrm{p}=0.7347)$. In total there was no clinical improvement in 5 (16.6\%) patients. Among the 11 patients who underwent curative surgery, 1 (9\%) died during hospital stay (due to disseminated intravascular coagulation) and 2 (18.1\%) came with features of recurrence/metastasis ( 1 each). At the end of 1 year follow-up 8 out of 11 (72.7\%) patients were disease free with improved quality of life. (table 2) Postoperative complications were wound infection (18\%), bleeding $(9 \%)$ and delayed gastric emptying (80\%) and could be managed conservatively save for one patient in which we had to reoperate for a bleed from the staple line of the gastrojejunostomy (the patient recovered thereafter).

Among the 19 patients given palliative treatment, 5 (26.3\%) died during hospital stay and 9 (47.3\%) during follow-up. At the end of 1 year only 5 (26.3\%) patients were surviving with improved quality of life and all of them were those who underwent surgical internal bypass. None of the patients who underwent either chemotherapy or PTBD and chemotherapy survived till 1 year. So mortality within 1 year was significantly lesser with curative than palliative treatment $(\mathrm{p}=0.0024)$. (table 3)

\section{DISCUSSION}

More than $60 \%$ of our patients were between $41-60$ years, which is in accordance with the study conducted by $\mathrm{CK}$ Yap, ${ }^{[31]}$ where the peak age distribution was between 50 to 60 yrs. (40\%). Our age distribution was between 28 and 72 yrs. (mean 36 years). This matches the study by Chun-Nan Yeh MD,[32] where the age range was between 29 to 89 yrs. (mean 59.0 yrs.). Slightly different results were noted in the study conducted by American Cancer Society where the average ages were 70 years and 72 years for IH-CCA and EHCCA respectively. ${ }^{[33]}$ In our study the female:male ratio was 1.5:1. However Sean F Altekruse [34] found a male to female ratio of 1.4 to 1 (IH-CCA) and 1.5 to 1 (EH-CCA). CK Yap ${ }^{[31]}$ found a male predominance (12:5).

In the existing literature, progressive jaundice is seen in $75-90 \%$ of patients. Abdominal pain, weight loss, pruritus occur in one third of patients or fewer. [3] This is similar to our findings (jaundice in 90\%, pain abdomen in 33.3\%). Our study also resembles that of Boris Blechacz who found IHCCA presents with nonspecific symptoms like abdominal pain, malaise, night sweats and cachexia. In a study conducted by Ahmad Ramzi Yusoff,[35] of the 69 patients diagnosed with CCA, 12 (17\%) had intrahepatic, 38 (55\%) perihilar and 19 (28\%) distal tumours. In our study EH-CCA was commoner. However, IH-CCA is on the rise.[3] In a study conducted by Renumathy Dhanasekaran and Alan W. Hemming,[36] 50.5\% patients underwent resection, 49.5\% were unresectable and received chemoradiotherapy, TACE or supportive care alone. The increase in percentage of resection was due to the availability of advanced surgical options, unlike our setup.

In a study conducted by William R. Jarnagin [37] from March 1991 through December 2000, 225 patients were evaluated. 32 of the 80 patients who underwent resection were alive at a median follow-up of 21 months; 27 remained disease free and 5 had recurrence. of the 9 actual 5-year survivors, 3 were alive without disease (median follow-up 88 months) and 6 died of disease recurrence and progression (median follow-up 77 months). Most studies had procedures like liver resection and transplantation. Despite these drawbacks and comparatively less curative surgery in our 
study, $72.7 \%$ of those who underwent curative surgery were disease free at 1 year.

In our study 5 of 6 patients (83\%) treated with palliative surgical bypass were surviving till 1 year with reasonable palliation with improved quality of life. Kuvshinoff BW et $\mathrm{al}^{[38]}$ treated 12 patients with irresectable or recurrent $\mathrm{p}$-CCA with internal biliary drainage followed by intraluminal (iridium-192) radiotherapy and EBRT. He reported a median survival of 14.5 months, all survived at least 6 months. Early complications were cholangitis $(n=2)$ and transient haemobilia $(n=1)$. Jaundice was relieved in 10 , while episodes of cholangitis were seen in 11 . The outcome of our study is comparable with this study, though in our study patients were provided only with chemotherapy post-surgical bypass.

Kubicka $S$ et al [39] investigated the effect of gemcitabine in 23 patients with nonresectable CCA in a phase II study. 7 had clinical benefit defined as a relief of tumour symptoms or gain of weight. But those who underwent palliative medical care $(n=13,43.3 \%)$ died within the follow up period with none surviving beyond 6 months in our study.

Palliative medical care is an ongoing area of research with recent additions like radiofrequency ablation, photodynamic therapy etc. Endoscopic biliary drainage is the other option which provides comparable outcome in terms of palliation and lesser complications as compared with PTBD, but is yet to be available in our rural tertiary setup.

\section{CONCLUSIONS}

CCA is not very uncommon and can be reasonably managed even at a rural tertiary care centre, though we can do better if liver resection devices like CUSA are available. However, our study is limited by a short duration and small sample size.

\section{ACKNOWLEDGEMENT}

We are extremely grateful to our patients and their families.

\section{REFERENCES}

[1] Bergquist A, Von Seth E. Epidemiology of cholangiocarcinoma. Best Pract Res Clin Gastroenterol 2015;29 (2):221-32.

[2] Plentz RR, Malek NP. Clinical presentation, risk factors and staging systems of cholangiocarcinoma. Best Pract Res Clin Gastroenterol 2015;29 (2):245-52.

[3] House MG, D'Angelica MI, Jarnaghin WR. Cancer of the bile ducts: extrahepatic biliary tumours. In: Jarnagin WR, edr. Blumgart's Surgery of the Liver, Biliary tract and pancreas. $6^{\text {th }}$ edn. Philadelphia: Elsevier 2012: p. 771-3.

[4] Blechacz B, Komuta M, Roskams T, et al. Clinical diagnosis and staging of cholangiocarcinoma. Nat Rev Gastroenterol Hepatol 2011;8 (9):512-22.

[5] Everhart JE, Ruhl CE. Burden of digestive diseases in the United States part III: liver, biliary tract and pancreas. Gastroenterology 2009;136 (4):1134-44.
[6] Mosadeghi S, Liu B, Bhuket T, et al. Sex-specific and race/ethnicity-specific disparities in cholangiocarcinoma incidence and prevalence in the USA: an updated analysis of the 2000-2011 Surveillance, Epidemiology and End Results registry. Hepatol Res 2016;46 (7):66977.

[7] Sriamporn S, Pisani P, Pipitgool V, et al. Prevalence of opisthorchis viverrini infection and incidence of cholangiocarcinoma in KhonKaen, Northeast Thailand. Trop Med Int Health 2004;9 (5):588-94.

[8] Koea J. Cancer of the bile ducts: intrahepatic cholangiocarcinoma. In: Jarnagin WR, edr. Blumgart's Surgery of the Liver, Biliary tract and pancreas. $6^{\text {th }}$ edn. Philadelphia: Elsevier 2012: p. 760.

[9] Roa JC, Adsay NV, Arola J, et al. Carcinoma of extrahepatic bile ducts. In: Klimstra DS, Lam AK, Paradis $\mathrm{V}$, et al. eds. tumours of the gallbladder and extrahepatic bile ducts. Digestive system tumours. WHO classification of tumours. $5^{\text {th }}$ edn. Lyon: International Agency for Research on Cancer 2019: p. 289-91.

[10] Endo I, Gonen M, Yopp AC, et al. Intrahepatic cholangiocarcinoma: rising frequency, improved survival and determinants of outcome after resection. Ann Surg 2008;248 (1):84-96.

[11] Weber SM, Jarnagin WR, Klimstra D, et al. Intrahepatic cholangiocarcinoma: resectability, recurrence pattern and outcomes. J Am Coll Surg 2001;193 (4):384-91.

[12] Sugiura T, Nishio $H$, Nagino $M$, et al. Value of multidetector-row computed tomography in diagnosis of portal vein invasion by perihilar cholangiocarcinoma. World J Surg 2008;32 (7):1478-84.

[13] Vogl TJ, Schwarz WO, Heller M, et al. Staging of Klatskin tumours (hilar cholangiocarcinomas): comparison of MR cholangiography, MR Imaging and endoscopic retrograde cholangiography. Eur Radiol 2006;16 (10):2317-25

[14] House MG, D'Angelica MI, Jarnaghin WR. Cancer of the bile ducts: extrahepatic biliary tumours. In: Jarnagin WR, edr. Blumgart's Surgery of the Liver, Biliary tract and pancreas. $6^{\text {th }}$ edn. Philadelphia: Elsevier 2012: p. 777.

[15] Tao LY, Cai L, He XD, et al. Comparison of serum tumour markers for intrahepatic cholangiocarcinoma and hepatocellular carcinoma. Am Surg 2010;76 (11):1210-3.

[16] Farges O, Fuks D, Le Treut YP, et al. AJCC 7th edition of TNM staging accurately discriminates outcomes of patients with resectable intrahepatic cholangiocarcinoma: Bby the AFC-IHCC-2009 study group. Cancer 2011;117 (10):2170-7.

[17] Deoliveira ML, Schulick RD, Nimura Y, et al. New staging system and a registry for perihilar cholangiocarcinoma. Hepatology 2011;53 (4):1363-71.

[18] Hong SM, Pawlik TM, Cho H, et al. Depth of tumour invasion better predicts prognosis than the current American Joint Committee on Cancer T classification for distal bile duct carcinoma. Surgery 2009;146 (2):250-7.

[19] Nagino M, Ebata T, Yokoyama Y, et al. Evolution of surgical treatment for perihilar cholangiocarcinoma: a single-center 34-year review of 574 consecutive resections. Ann Surg 2013;258 (1):129-40.

[20] Ebata T, Yokoyama Y, Igami T, et al. Hepatopancreatoduodenectomy for cholangiocarcinoma: 
a single-center review of 85 consecutive patients. Ann Surg 2012;256 (2):297-305.

[21] Morimoto Y, Tanaka Y, Ito T, et al. Long-term survival and prognostic factors in the surgical treatment for intrahepatic cholangiocarcinoma. J Hepatobiliary Pancreat Surg 2003;10 (6):432-40.

[22] Konstadoulakis MM, Roayaie S, Gomatos IP, et al. Fifteenyear, single-center experience with the surgical management of intrahepatic cholangiocarcinoma: operative results and long-term outcome. Surgery 2008;143 (3):366-74.

[23] Lee SG, Song GW, Hwang S, et al. Surgical treatment of hilar cholangiocarcinoma in the new era: the Asan experience. J Hepatobiliary Pancreat Sci 2010;17 (4):476-89.

[24] Van der Gaag NA, Rauws EAJ, Van Eijck CHJ, et al. Preoperative biliary drainage for cancer of the head of the pancreas. N Engl J Med 2010;362 (2):129-37.

[25] Sewnath ME, Karsten TM, Prins MH, et al. A metaanalysis on the efficacy of preoperative biliary drainage for tumours causing obstructive jaundice. Ann Surg 2002;236 (1):17-27.

[26] Choi SB, Kim KS, Choi JY, et al. The prognosis and survival outcome of intrahepatic cholangiocarcinoma following surgical resection: association of lymph node metastasis and lymph node dissection with survival. Ann Surg Oncol 2009;16 (11):3048-56.

[27] Aoba T, Ebata T, Yokoyama Y, et al. Assessment of nodal status for perihilar cholangiocarcinoma: location, number or ratio of involved nodes. Ann Surg 2013;257 (4):718-25.

[28] Yoshida T, Matsumoto T, Sasaki A, et al. Prognostic factors after pancreatoduodenectomy with extended lymphadenectomy for distal bile duct cancer. Arch Surg 2002;137 (1):69-73.

[29] Sakamoto Y, Kosuge T, Shimada K, et al. Prognostic factors of surgical resection in middle and distal bile duct cancer: an analysis of 55 patients concerning the significance of ductal and radial margins. Surgery 2005;137 (4):396-402.
[30] National Comprehensive Cancer Network (NCCN) NCCN clinical practice guidelines in oncology: hepatobiliary cancers. Fort Washington: NCCN, c2016. [cited 2015 Nov 5]. http://www.nccn.org/professionals/physician_gls/pdf/ hepatobiliary.pdf.

[31] Yap CK, Chee EN, Consigliere D, et al. Four year experience with cholangiocarcinoma: a survey of patients, clinical presentation, management and prognosis in two hospitals. Singapore Med J 1992;33 (3):235-8.

[32] Yeh CN, Jan YY, Chen MF. Influence of age on surgical treatment of peripheral cholangiocarcinoma, Am J Surg 2004;187 (4):559-63.

[33] https://amp.cancer.org/cancer/bile-ductcancer/about/key.statistics.html.

[34] Altekruse SF, Petrick JL, Rolin AI, et al. Geographic variation of intrahepatic cholangiocarcinoma, extrahepatic cholangiocarcinoma, and hepatocellular carcinoma in the United States. PLoS One 2015;10 (3):e0120574.

[35] Yusoff AR, Razak MMA, Yoong BK, et al. Survival analysis of cholangiocarcinoma: a 10-year experience in Malaysia. World J Gastroenterol 2012;18 (5):458-65.

[36] Dhanasekaran R, Hemming AW, Zendejas I, et al. Treatment outcomes and prognostic factors of intrahepatic cholangiocarcinoma. Oncol Rep 2013;29 (4):1259-67.

[37] Jarnagin WR, Burke E, Powers C, et al. Intrahepatic biliary enteric bypass provides effective palliation in selected patients with malignant obstruction at the hepatic duct confluence. Am J Surg 1998;175 (6):453-60.

[38] Kuvshinoff BW, Armstrong JG, Fong Y, et al. Palliation of irresectable hilar cholangiocarcinoma with biliary drainage and radiotherapy. Br J Surg 1995;82 (11):15225.

[39] Kubicka S, Rudolph KL, Tietze MK, et al. Phase II study of systemic gemcitabine chemotherapy for advanced unresectable hepatobiliary carcinomas. Hepatogastroenterology 2001;48 (39):783-9. 